\title{
Reproduction du Mugilidae Mugil cephalus Linné, 1758 dans deux complexes lagunaires (lagunes Ébrié et de Grand-Lahou) de la Côte d'Ivoire
}

\author{
E. Luc Gervais DJADJI ${ }^{1,2}$, Boua Célestin ATSE ${ }^{1 *}$, Soumaïla SYLLA ${ }^{1,2}$, \\ Justin Kouadio KONAN ${ }^{1,2}$ et Joël N'Guessan KOUASSI ${ }^{2}$ \\ ${ }^{1}$ Centre de Recherches Océanologiques (CRO), B.P.V 18 Abidjan, Fax (225) 213511 55, Côte d'Ivoire. \\ ${ }^{2}$ Laboratoire d'Hydrobiologie, Université de Cocody (Abidjan, Côte d'Ivoire), UFR Biosciences, \\ 04 B.P. 322 Abidjan 04, Côte d'Ivoire. \\ *Auteur correspondant,E-mail : atse_boua_celestin@hotmail.com
}

\section{RESUME}

La reproduction de Mugil cephalus a été étudiée dans les lagunes Ébrié et de Grand-Lahou de janvier 2006 à décembre 2007. Au total, 2041 individus ont été échantillonnés. Immédiatement, après la capture, les longueurs à la fourche ont été mesurées au millimètre près et les masses totales et éviscérées l'ont été à $0,1 \mathrm{~g}$ près. Les gonades et le foie ont été prélevés et pesés à $0,001 \mathrm{~g}$ près. Les périodes de ponte ont été établies en analysant l'évolution temporelle de la fréquence de maturité sexuelle, des variations mensuelles des indices gonadosomatique et hepatosomatique et du facteur de condition. La sex-ratio a été établie en fonction de la taille des individus. Les tailles de première maturité sexuelle ont été estimées au moyen de fonction logistique. Les sex-ratios de 1:0,20 (lagune Ébrié) et de 1:0,51 (lagune de Grand-Lahou) sont en faveur des mâles. Les mâles atteignent la maturité sexuelle à une taille inférieure aux femelles. La période d'émission des gamètes s'étend d'octobre à janvier et Mugil cephalus ne réalise pas de ponte en lagune. La fécondité absolue varie de 126681 à 2844890 ovocytes.

(c) 2013 International Formulae Group. All rights reserved.

Mots clés : Mugil cephalus, sex-ratio, fécondité, période de ponte.

\section{INTRODUCTION}

Les Mugilidae sont des poissons côtiers des mers tropicales et tempérées. Les milieux saumâtres estuariens et lagunaires constituent leur milieu de vie; domaine de prédilection (Albaret, 1992). Les travaux de Séret et Opic (1986) ont permis de connaître la systématique de ces poissons. En Afrique de l'Ouest, les Mugilidae sont représentés par les genres Mugil et Liza. En Côte d'Ivoire, les travaux réalisés sur la famille des Mugilidae sont ceux d'Albaret et Legendre (1985). Il ressort de ces travaux que, les mulets font souvent l'objet de pêcheries spécialisées et sont inégalement repartis dans les trois systèmes lagunaires Aby, Ébrié et GrandLahou. Parmi les espèces rencontrées, seules, Mugil cephalus et Liza falcipinnis présentent un intérêt clairement établi pour l'élevage (Albaret et Legendre, 1985). Par ailleurs, Mugil cephalus est élevé avec succès dans diverses régions du monde depuis de nombreuses années notamment en Asie (Japon, Taïwan et Hawaï), en Europe (Italie) et en Afrique (Egypte et Tunisie) (Magdy, 2004 ; Meseda et Samira, 2006). L'élevage de 
cette espèce passe par la connaissance de son écologie alimentaire et de sa biologie de reproduction en milieu naturel. Peu d'informations sont disponibles en ce qui concerne la biologie et l'écologie des Mugilidae de l'Afrique de l'Ouest en général et en Côte d'Ivoire en particulier.

La présente étude se propose de déterminer la biologie de la reproduction de Mugil cephalus dans les lagunes Ébrié et de Grand-Lahou à travers la connaissance de la sex-ratio, de la taille de première maturité, de la fécondité et de la période de reproduction.

\section{MATERIEL ET METHODES \\ Milieu d'étude}

En Côte d'Ivoire, les lagunes (Aby, Ébrié, Grand-Lahou) sont situées le long de la moitié orientale de la façade littorale et couvrent une superficie d'environ $1200 \mathrm{~km}^{2}$. Elles bénéficient d'un climat de type équatorial de transition avec deux grandes saisons (pluvieuse d'avril à juillet et sèche de décembre à mars) et deux petites saisons (pluvieuse d'octobre à novembre et sèche d'août à septembre). Les lagunes Ébrié et de Grand-Lahou (Figure 1) s'étirent d'Est en Ouest respectivement sur $130 \mathrm{~km}$ et $50 \mathrm{~km}$ avec des superficies respectives de $566 \mathrm{~km}^{2}$ et 190 km² (Durand et Guiral, 1994). Le système lagunaire Ebrié qui est en relation permanente avec l'Océan Atlantique par l'intermédiaire du Canal de Vridi, est un milieu peu profond $(4,8$ $\mathrm{m}$ en moyenne). Sur l'ensemble de la lagune, la salinité est comprise entre 0 et $30 \%$. Les températures moyennes sont élevées mais présentent de faibles variations saisonnières avec un maximum de $31,2{ }^{\circ} \mathrm{C}$ mesuré en surface en avril et un minimum de $27,4{ }^{\circ} \mathrm{C}$ en août (Durand et Guiral, 1994). Le système lagunaire de Grand-Lahou a une profondeur moyenne de $3 \mathrm{~m}$. Tenant compte de la salinité, la lagune de Grand-Lahou peut être divisée en deux régions distinctes : la région continentale de salinité allant de 0 à $19 \%$ et la région océanique plus salée avec des valeurs comprises entre $8 \%$ et $27 \%$. Elle est moins renouvelée. Les eaux restent toujours saumâtres parce qu'elles sont moins influencées par les apports continentaux
(Konan et al., 2008). La température moyenne des eaux de la lagune varie de $25^{\circ} \mathrm{C}$ à $28{ }^{\circ} \mathrm{C}$. Les valeurs maximales de $30^{\circ} \mathrm{C}$ à $31{ }^{\circ} \mathrm{C}$ ont été observées en mars et en avril pendant la grande saison sèche et la valeur minimale de $17{ }^{\circ} \mathrm{C}$ en août pendant la petite saison sèche en raison de l'upwelling côtier (Konan et al., 2008).

\section{Echantillonnage et mesure des paramètres de reproduction}

L'échantillonnage ichtyologique a été réalisé mensuellement (de janvier 2006 à décembre 2007). Trois batteries de filets maillants de différentes mailles 10, 12, 15, 20, $25,30,35,40$ et $50 \mathrm{~mm}$ ont été utilisées. Les pêches ont été effectuées de nuit et de jour. Les filets ont été posés à $17 \mathrm{~h}$ et visités le lendemain à $7 \mathrm{~h}$ pour la pêche de nuit puis, ils sont immédiatement reposés à nouveau et relevés à $13 \mathrm{~h}$ pour la pêche de jour. (Ntakimazi et al., 2000). Les poissons capturés ont été identifiés avec la clé de détermination d'Albaret (1992), conservés sous glace sèche et transportés immédiatement à la Station d'Aquaculture Expérimentale de Layo pour les mesures. Sur chaque individu capturé, la longueur à la fourche a été mesurée $\mathrm{au} \mathrm{cm}$ près. Les masses totales et éviscérées ont été déterminées avec une balance de type Sartorius (modèle LP 6200S) de précision $0,01 \mathrm{~g}$ et de portée $6200 \mathrm{~g}$. Les masses des gonades et du foie ont été pesées grâce à une balance Sartorius (modèle BP 221S) de précision $0,0001 \mathrm{~g}$ près et de portée $220 \mathrm{~g}$. Le sexe a été déterminé par examen macroscopique des gonades. La détermination des stades de maturité sexuelle a été faite sur la base de l'échelle de maturité sexuelle d'Ameur et al. (2003) que nous avons modifiée en fonction des observations faites sur le terrain:

Stade 1: les gonades sont très fines, transparentes et disposées en $\mathrm{V}$ dont la pointe se situe à l'extrémité postérieure de la cavité générale ;

Stade 2: les gonades se présentent sous forme de fins filaments blanchâtres. Chaque gonade est longée par de fins vaisseaux sanguins nettement visibles. La gonade ne 
dépasse pas le quart antérieur de la cavité abdominale ;

Stade 3 : c'est le début de maturation. Les testicules sont blancs à section aplatie et les ovaires ont une couleur rose claire à section ovale. Les ovocytes ne sont pas visibles à l'œil nu ;

Stade 4 : c'est la maturation. Les gonades sont bien développées et occupent une grande partie de la cavité abdominale. Les testicules sont blancs et vascularisés. Les ovaires sont de couleur jaune peu orangé à section arrondie avec de petits ovocytes visibles à l'œil nu ;

Stade 5: c'est la maturation avancée. Les gonades remplissent la majeure partie de la cavité abdominale. La moindre pression sur l'abdomen fait couler la laitance chez les mâles et les ovocytes chez les femelles ;

Stade 6: c'est la poste ponte. Les gonades perdent leur fermeté. Chez le mâle, la gonade est d'un aspect sanguinolent. Chez la femelle, les ovocytes paraissent comme dispersées dans l'ovaire.

La taille de première maturité sexuelle a été déterminée pour les mâles et les femelles en considérant comme matures les poissons présentant des gonades au stade supérieur ou égal à 4. La proportion d'individus matures (P), par classe de taille de $1 \mathrm{~cm}$, a été ajustée par la fonction de Ghorbel et al. (1996) selon l'équation :

$\mathrm{P}=\frac{1}{1+\mathrm{e}^{-(\mathrm{a}+\mathrm{bLF})}}$ avec $\mathrm{P}=$ proportion

d'individus matures; $L F=$ longueur à la fourche ;

$\mathrm{a}$ et $\mathrm{b}=$ constantes. De cette formule, nous avons déduit les tailles à $\mathrm{LF}_{50}$ (Ghorbel et al., 1996) en calculant le rapport suivant:

$\mathrm{LF}_{50}=-\frac{a}{b}$.

L'indice gonado-somatique (IGS) et l'indice hépato-somatique (IHS) des individus matures ont été calculés mensuellement à partir des formules suivantes :

$$
\begin{aligned}
& \text { IGS }=\frac{M \quad g}{M \text { év }} \times 100 \quad \text { et } \\
& \text { IHS }=\frac{M_{f}}{M_{\text {év }}} \times 100 ; \text { avec } M_{g}=\text { masse des }
\end{aligned}
$$

gonades $(\mathrm{g}) ; \mathrm{M}_{\mathrm{év}}=$ masse éviscérée $(\mathrm{g})$ et $\mathrm{M}_{\mathrm{f}}$ $=$ masse $\mathrm{du}$ foie $(\mathrm{g})$. Pour vérifier le rôle éventuel des réserves musculaires dans la gamétogénèse, le facteur de condition $(\mathrm{K})$ a été déterminé selon la formule : $\mathrm{K}=\frac{\mathrm{M}_{\text {év }}}{\mathrm{LF}^{3}} \times 100 ;$ avec $\mathrm{M}_{\mathrm{ev}}=$ masse éviscérée $(\mathrm{g})$ et $\mathrm{LF}=$ longueur à la fourche (cm). Les variations mensuelles des indices gonado-somatique (IGS) et hépato-somatique (IHS) ont été suivies pour décrire le cycle sexuel et déterminer la période de ponte. La fécondité absolue a été déterminée par comptage des ovocytes des ovaires au stade 4. Les diamètres des ovocytes ont été mesurés à la loupe binoculaire munie d'un micromètre (model Wild M3C). Les diamètres ovocytaires ont été mesurés estimé à partir de mensurations faites sur 30 ovocytes par femelle. La sex-ratio $(\mathrm{M} / \mathrm{F})$ a été calculée à partir d'individus de sexe connu. La sex-ratio a été déterminée en fonction des tailles groupées par classe de $1 \mathrm{~cm}$ de longueur à la fourche.

Les relations fécondité absolue longueur à la fourche et fécondité absolue masse totale ont été décrites respectivement par les équations suivantes : $\mathrm{F}=\mathrm{a} \mathrm{LF}^{\mathrm{b}}$ et $\mathrm{F}=\mathrm{a} \mathrm{M}_{\mathrm{t}}^{\mathrm{b}}$ avec

$\mathrm{F}=$ fécondité absolue $; \mathrm{LF}=$ longueur à la fourche $(\mathrm{cm}) ; \mathrm{M}_{\mathrm{t}}=$ masse totale du poisson en (g) ;

$\mathrm{b}=$ pente et $\mathrm{a}=$ ordonnée à l'origine.

\section{Analyse statistique}

Les pourcentages des sexes et les tailles de première maturité ont été comparés au moyen du test du Chi-deux. L'analyse de variance à un facteur a été utilisée pour déterminer les effets des saisons (saison sèche et saison pluvieuse) et des années (première et deuxième année) sur l'IGS, l'IHS et le K en fonction du sexe. Le test de Tukey a été utilisé pour les comparaisons a posteriori lorsque les effets sont significatifs. Les différences sont considérées significatives à $\mathrm{p}<0,05$. Les analyses ont été effectuées avec le logiciel STATISTICA 7.1. 


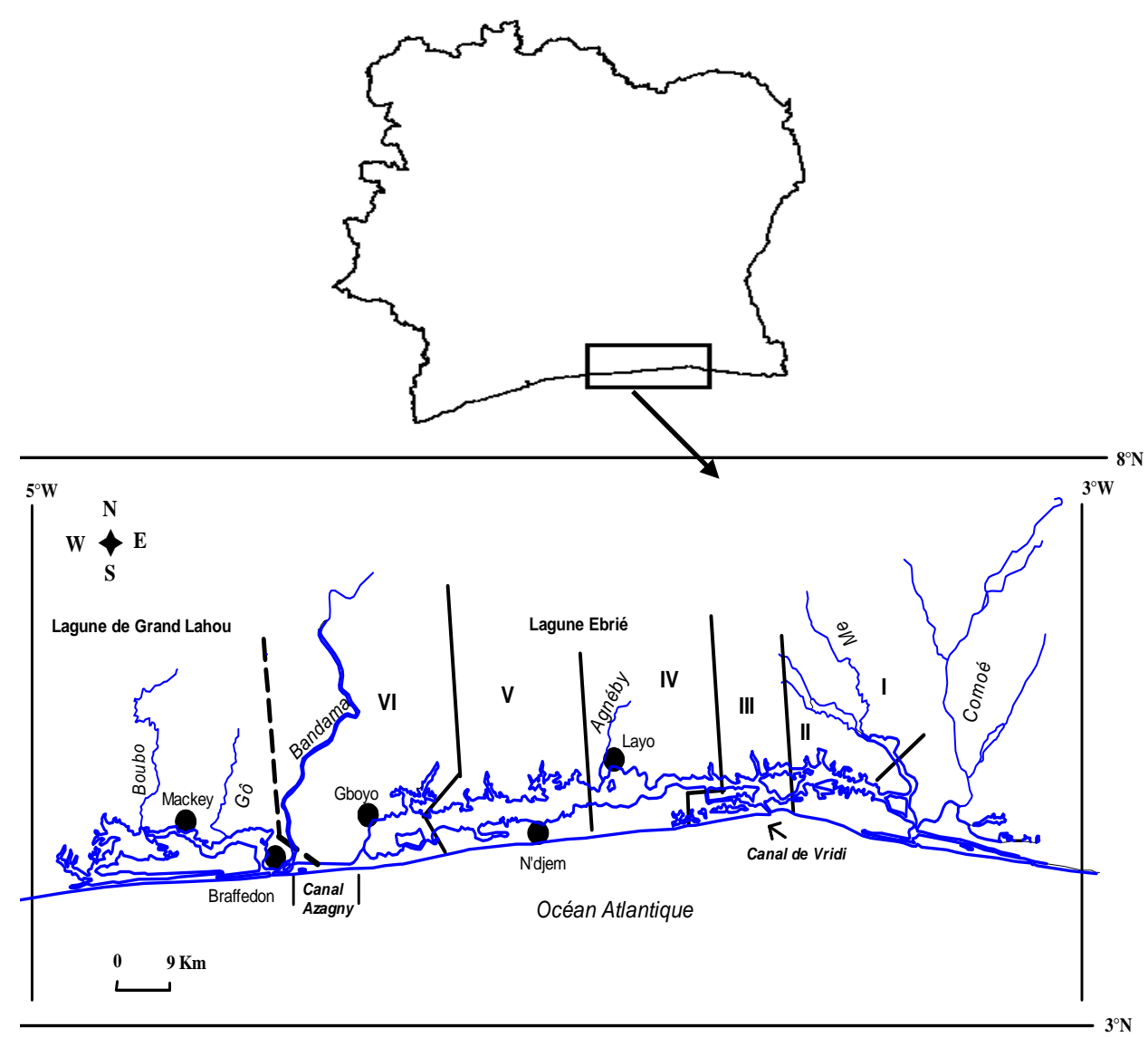

Figure 1: Situation géographique des lagunes Ébrié et de Grand-Lahou (Côte d'Ivoire) et localisation des stations d'échantillonnage $(\bullet)$.

\section{RESULTATS}

\section{Sex-ratio}

Au total, 1526 individus (1266 mâles et 260 femelles) ont été examinés en lagune Ébrié contre 515 dans la lagune de GrandLahou (341 mâles et 174 femelles). Les sexratios obtenues $(1: 0,20)$ et $(1: 0,51)$ respectivement dans les lagunes Ebrié $\left(\chi^{2}=\right.$ $663,19 ; \mathrm{p}<0,05)$ et Grand-Lahou $\left(\chi^{2}=54,15\right.$ $; \mathrm{p}<0,05)$ sont significativement différentes de la sex-ratio théorique $1: 1$. Elle est en faveur des mâles dans chacune des lagunes (Tableau 1). La sex-ratio en fonction de la taille révèle qu'en lagune Ebrié (Figure $2 \mathrm{~A}$ ), les individus de taille comprise entre 16,5 et $18,5 \mathrm{~cm}$ (LF) sont exclusivement dominés par les mâles. A partir de cette taille, on note un nombre assez élevé de femelles. La distribution de la sex-ratio est à dominance mâle pour les individus de taille comprise entre 45,5 et $50,5 \mathrm{~cm}$ (LF). Au delà, la distribution est essentiellement femelle. Dans la lagune de Grand-Lahou (Figure 2B), les classes de tailles de 19,5 à $25,5 \mathrm{~cm}$ sont essentiellement dominées par les femelles. Entre 26 et $43,5 \mathrm{~cm}$, la sex-ratio est à dominance mâles avec parfois des présences exclusives à certaines tailles (26 et $27,5 \mathrm{~cm}$ et entre 43,5 et $45,5 \mathrm{~cm}$ (LF). A partir de 48,5 $\mathrm{cm}$, la sex-ratio est à dominance exclusive femelles.

\section{Taille de première maturité sexuelle}

La taille de première maturité sexuelle $\left(\mathrm{LF}_{50}\right)$ est de 30,3 cm chez les mâles et de 32,9 $\mathrm{cm}$ chez les femelles en lagune Ébrié (Figure 
3). En revanche, elle est de $32,9 \mathrm{~cm}$ chez les mâles et de $35,2 \mathrm{~cm}$ chez les femelles en lagune de Grand-Lahou. Aucune différence significative de la taille de première maturité entre les mâles et les femelles n'a été observée en lagune Ebrié $\left(\chi^{2}=0,1 ; p>0,05\right)$ et en lagune de Grand-Lahou ( $\left.\chi^{2}=0,08 ; p>0,05\right)$.

Evolution saisonnière des indices gonadosomatique (IGS), hépato-somatique (IHS) et du facteur de condition (K)

D'une année à l'autre, la variation mensuelle de l'IGS, de l'IHS et du K des mâles et des femelles n'a révélé aucune différence significative, quelle que soit la lagune. Par conséquent, les données des deux années ont été fusionnées par lagune. Dans la lagune Ébrié, l'IGS moyen mensuel des mâles et des femelles (Figure $4 \mathrm{~A} 1$ ) croit d'avril à octobre, où un pic est observé au niveau de chaque sexe. Il varie de $0,14 \pm 0,02$ à $0,66 \pm$ 0,09 chez les mâles et de $0,30 \pm 0,13$ à $5,59 \pm$ 0,59 chez les femelles. Par contre, l'IGS décroit de novembre à janvier quel que soit le sexe (respectivement de $0,34 \pm 0,02$ à $0,03 \pm$ 0,01 chez les mâles et de $3,07 \pm 0,25$ à $0,42 \pm$ 0,18 chez les femelles). Dans la lagune de Grand-Lahou (Figure 4 A2), l'IGS croit à partir de juillet pour atteindre un pic en octobre chez les deux sexes. Il s'étend de 0,13 $\pm 0,00$ à $0,36 \pm 0,06$ chez les mâles et de 1,93 $\pm 0,25$ à $5,09 \pm 0,40$ chez les femelles. Il décroît de novembre à avril et varie de $0,36 \pm$ 0,08 à $0,11 \pm 0,00$ chez les mâles et de 4,13 \pm 0,37 à $1,42 \pm 0,15$ chez les femelles. Quant à l'IHS, en lagune Ébrié (Figure 4 B1), il crô̂t de mars à août, où un pic est observé pour les deux sexes. Il évolue de $0,95 \pm 0,01$ à $1,19 \pm$ 0,03 chez les mâles et de $0,96 \pm 0,01$ à $1,23 \pm$ 0,03 chez les femelles. L'IHS décroît de septembre à octobre chez les deux sexes. Les valeurs moyennes varient de $0,96 \pm 0,02$ à $0,92 \pm 0,01$ chez les mâles et de $0,98 \pm 0,01$ à $0,94 \pm 0,01 \mathrm{chez}$ les femelles. En revanche, dans la lagune de Grand-Lahou (Figure 4 B2), l'IHS croit de mars à juillet, où un pic est atteint puis décroît d'août à octobre pour les deux sexes. Les valeurs moyennes de l'IHS pour la phase ascendante s'étendent de 0,93 \pm
0,01 à $1,01 \pm 0,03$ pour les mâles et de $1,09 \pm$ 0,06 à $1,25 \pm 0,05$ pour les femelles. Pour celle descendante, la valeur moyenne de l'IHS s'étale de $0,94 \pm 0,02$ à $0,89 \pm 0,01$ chez les mâles et de $0,95 \pm 0,06$ à $0,84 \pm 0,03$ chez les femelles. En ce qui concerne le facteur de condition (K) (Figure $4 \mathrm{C1}$ ), il croît de mai à octobre chez les mâles et d'avril à octobre chez les femelles en lagune Ébrié. Les valeurs moyennes du facteur $\mathrm{K}$ varient alors de 1,17 \pm 0,01 à $1,30 \pm 0,03$ chez les mâles et de 1,24 \pm 0,01 à $1,34 \pm 0,02$ chez les femelles. Il chute ensuite de novembre à février chez les deux sexes pour des moyennes de $1,28 \pm 0,03$ à $1,17 \pm 0,01$ chez les mâles et de $1,30 \pm 0,03$ à $1,22 \pm 0,01 \mathrm{chez}$ les femelles. Dans la lagune de Grand-Lahou (Figure 4C2), le facteur K croit de mai à octobre où il atteint un pic puis, décroît de novembre à avril chez les deux sexes. Les moyennes des valeurs s'étendent de $1,39 \pm 0,01$ à $1,43 \pm 0,01$ chez les mâles et de $1,38 \pm 0,01$ à $1,43 \pm 0,01$ chez les femelles en phase ascendante. Elles chutent ensuite de $1,41 \pm 0,01$ à $1,39 \pm 0,01$ chez les mâles puis, de $1,41 \pm 0,01$ à $1,38 \pm 0,01$ chez les femelles.

Variation mensuelle du pourcentage des stades de maturité sexuelle

Les individus mâles et femelles aux stades 4 et 6 ont été capturés dans les deux lagunes toute l'année (Figure 5). En revanche, aucun spécimen mâle et femelle au stade 5 n'a été observé dans les deux lagunes (Figure 5). La variation saisonnière du pourcentage de maturation sexuelle montre que les femelles aux stades 1, 3, 4 et 6 sont présentes toute l'année mais en proportions variables. En lagune Ebrié (Figure 5A1), chez les mâles, les stades 1, 3, 4 et 6 se rencontrent toute l'année à l'exception du stade 2 qui n'apparait qu'entre les mois de novembre et de décembre. Chez les femelles (Figure 5B1), les stades de maturation sexuelle 1,3 et 6 sont observés sur toute la période d'échantillonnage; ce qui n'est pas le cas des stades 2 et 4 . Les femelles au stade 4 se rencontrent d'août à octobre. Les femelles en période de repos sexuel (stade 6) se 
rencontrent de février à juin. En lagune de Grand-Lahou, chez les mâles (Figure 5A2), les stades 1, 3, 4 et 6 se répartissent sur toute l'année. En revanche, le stade 2 ne s'observe qu'entre juin et août. Les individus au stade 6 sont les plus fortement représentés. Les mois de février à juillet sont celles où se rencontre une forte concentration d'individus mâles aux stades 1, 3, 4 et 6 . Chez les femelles (Figure 5B2), les stades de maturation sexuelle 3 , 4 et 6 sont les plus dominantes. En revanche, dans cette lagune, les femelles aux stades 1 et 2 sont presqu'inexistantes. Contrairement à la lagune Ebrié, les femelles au stade de maturation sexuelle 4 sont fortement représentées. Les femelles en période de repos sexuelle (stade 6) s'observent également de février à juin. La période de ponte dans les lagunes Ebrié et de Grand-Lahou s'étale d'octobre à janvier.

\section{Diamètres ovocytaire et Fécondité}

$\mathrm{La}$ distribution des fréquences de diamètres ovocytaires chez les femelles aux stades 4 de maturité sexuelle est comprise entre 0,35 et $0,70 \mathrm{~mm}$ (avec une majorité d'ovocytes de taille comprise entre 0,55 et $0,60 \mathrm{~mm}$ ) quelle que soit la lagune (Figure 6). La fécondité absolue a été déterminée chez les femelles au stade 4. Elle varie de 135136 à 2 844890 ovocytes dans la lagune Ébrié pour des femelles de tailles comprises entre 20,1 et $62,3 \mathrm{~cm}$ (LF). Quant à la fécondité relative, la moyenne est de $801 \pm 394$ ovocytes.g ${ }^{-1}$ de masse corporelle. Dans la lagune de GrandLahou, la fécondité absolue varie de 126681 à 2789869 ovocytes pour des femelles de tailles variant entre 19,4 et 60,1 cm (LF). La fécondité relative moyenne est de $966 \pm 427$ ovocytes. $\mathrm{g}^{-1}$ de masse corporelle. La fécondité absolue est positivement corrélée à la longueur à la fourche et à la masse totale aussi bien en lagune Ébrié.

(respectivement $\mathrm{F}=46$ 648,86 x LF - 1,12 x $10^{6} ; \mathrm{r}^{2}=0,63$ et $\mathrm{P}<0,05$ et $\mathrm{F}=625,31 \times \mathrm{M}_{\mathrm{t}}+78969,23 ; \mathrm{r}^{2}=0,77$ et $\mathrm{P}<$ $0,05)$, qu'en lagune de Grand-lahou (respectivement $\mathrm{F}=52678,67 \times \mathrm{LF}-1,10 \mathrm{x}$ $10^{6} ; \mathrm{r}^{2}=0,55$ et $\mathrm{P}<0,05$ et $\mathrm{F}=654,06 \times \mathrm{M}_{\mathrm{t}}+$ $2,31 \times 10^{5} ; r^{2}=0,49$ et $\left.\mathrm{P}<0,05\right)$.

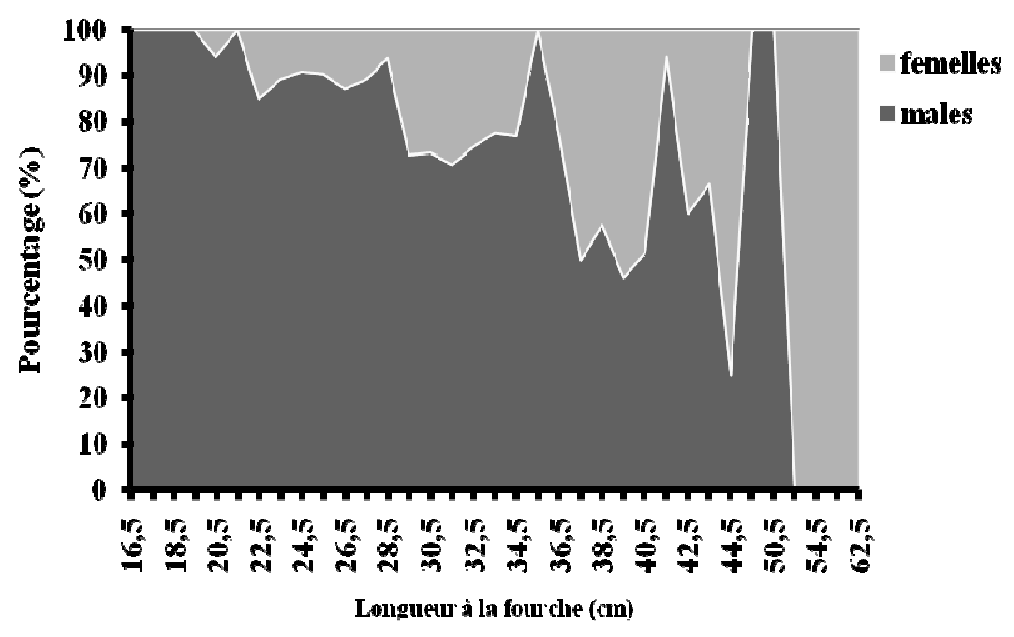

A ) Ebrié 


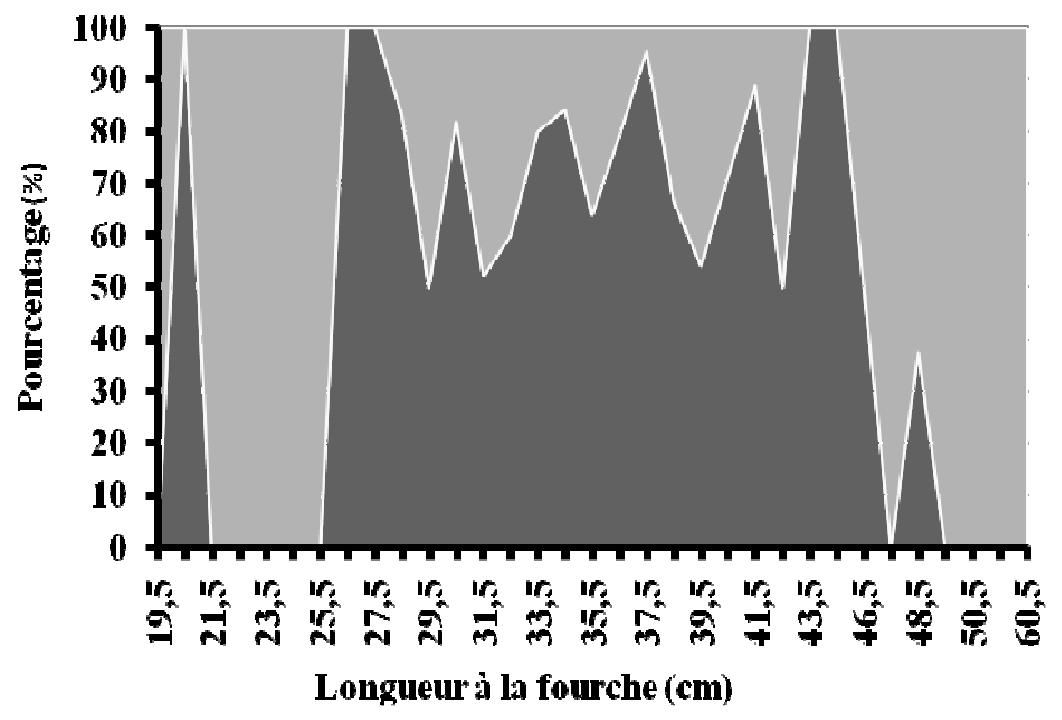

B ) Grand-Lahou

Figure 2: Sex-ratio en fonction de la longueur à la fourche chez Mugil cephalus dans les lagunes Ebrié (A) et de Grand-lahou (B).

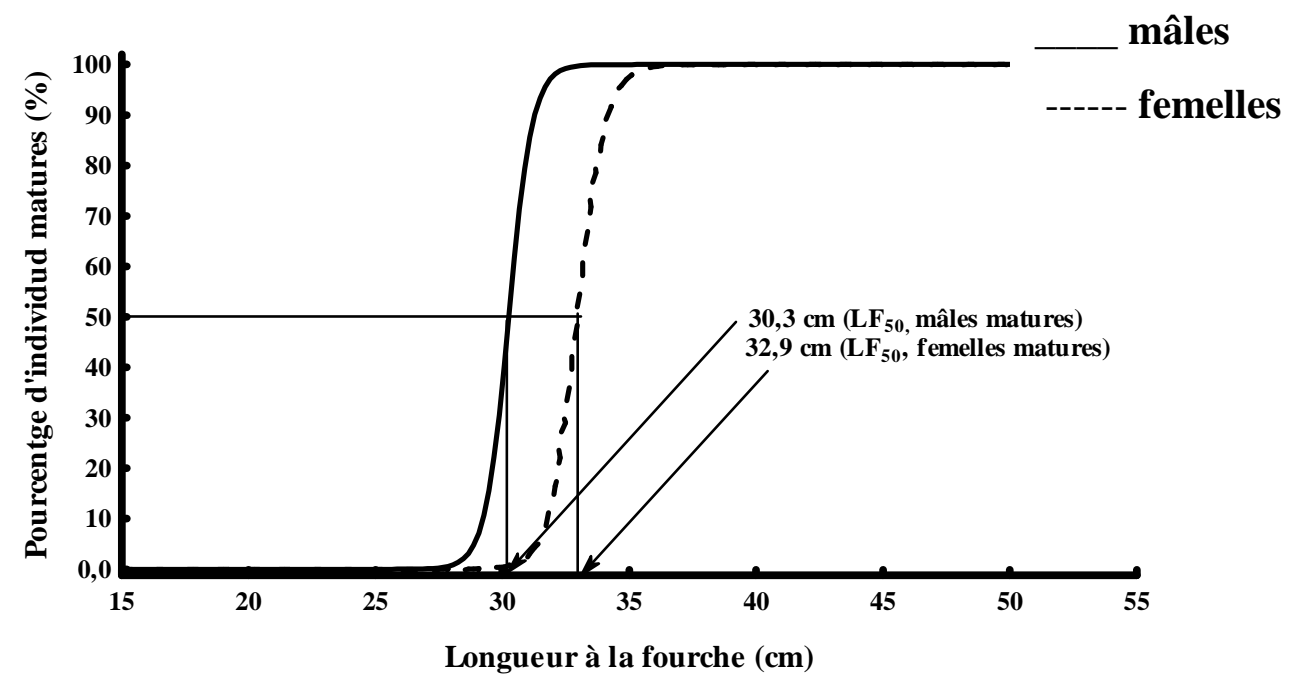

A ) Ebrié 


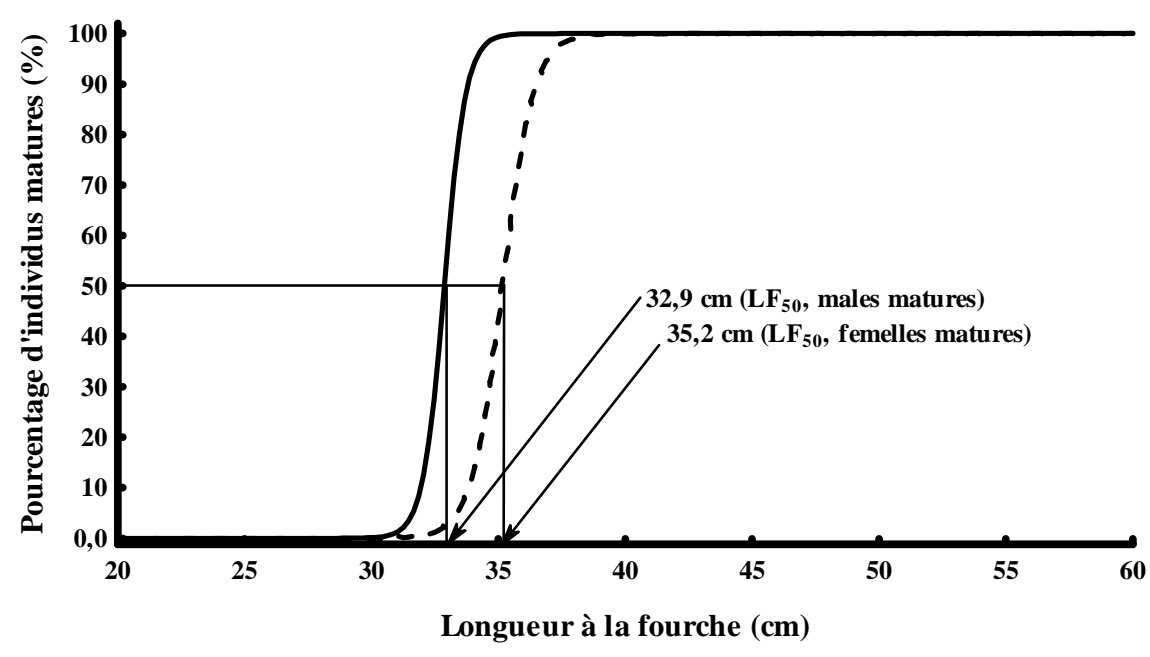

\section{B) Grand-Lahou}

Figure 3 : Courbe logistique pour la détermination de la taille de première maturité sexuelle $\left(\mathrm{L}_{50}\right)$ en fonction de la longueur à la fourche (LF) des mâles et des femelles de Mugil Cephalus dans les lagunes Ébrié (A) et de Grand Lahou (B).

Ebrié

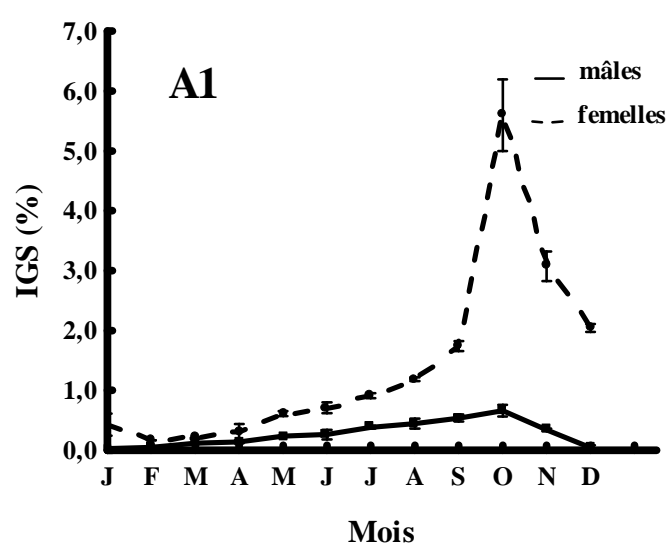

Grand -Lahou

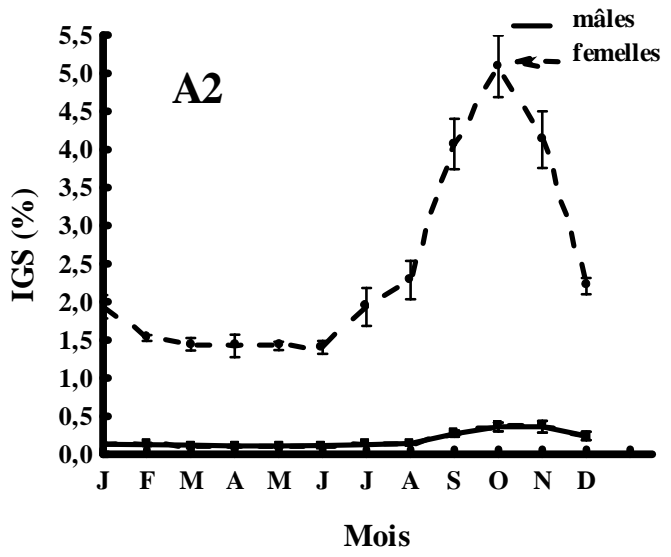



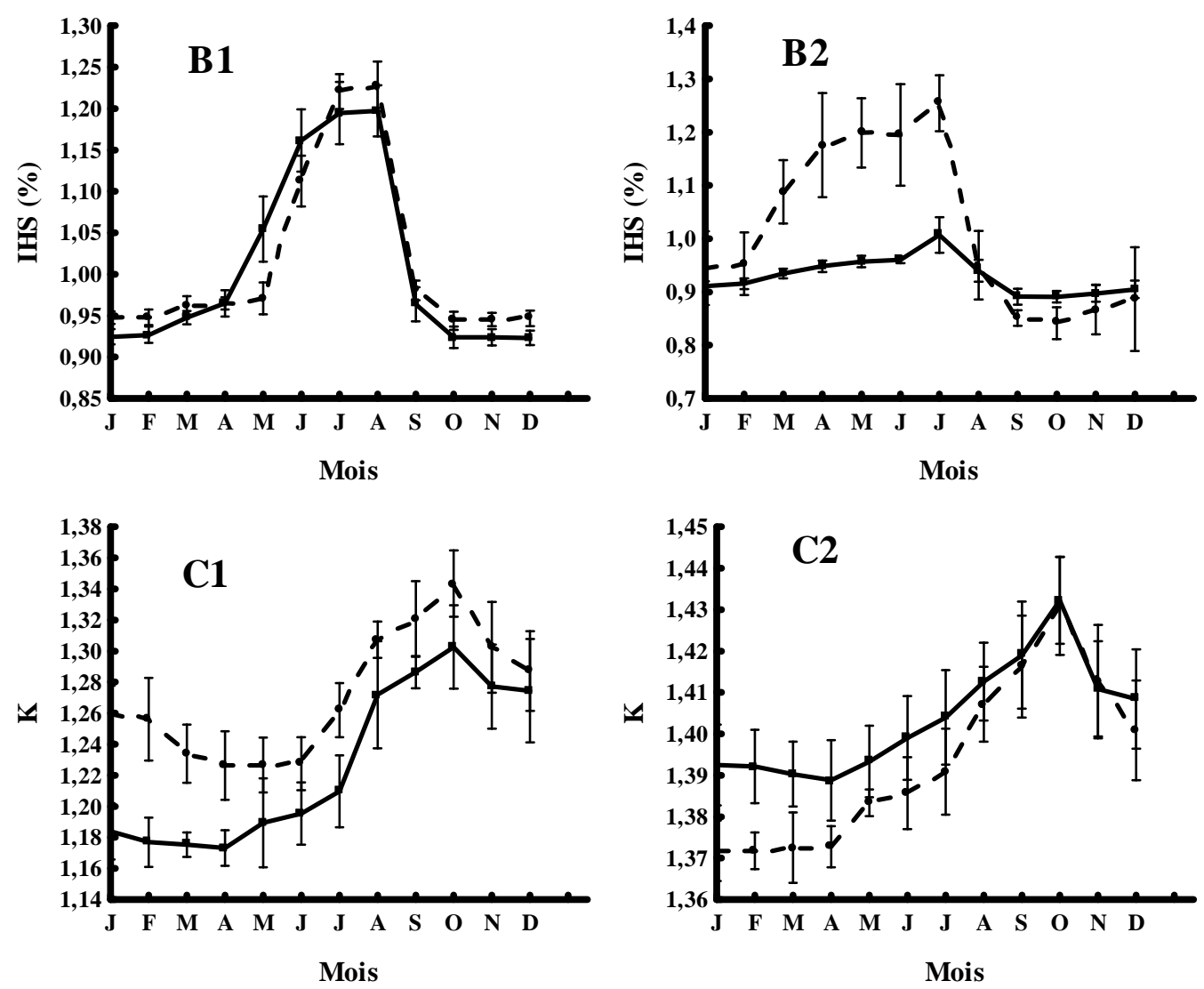

Figure 4 : Variations mensuelles de l'indice gonadosomatique (IGS), de l'indice hépatosomatique (IHS) et du facteur de condition (K) des mâles et des femelles de Mugil cephalus dans les lagunes Ébrié (A1, B1, C1) et de Grand-lahou (A2, B2, C2).

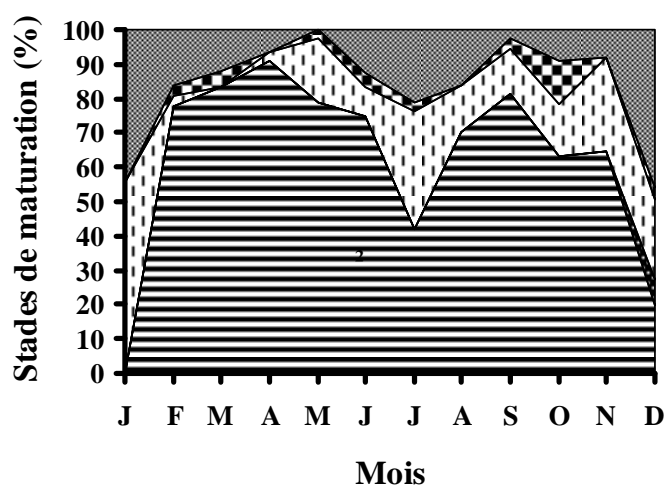

Ebrié : A1 Mâles 


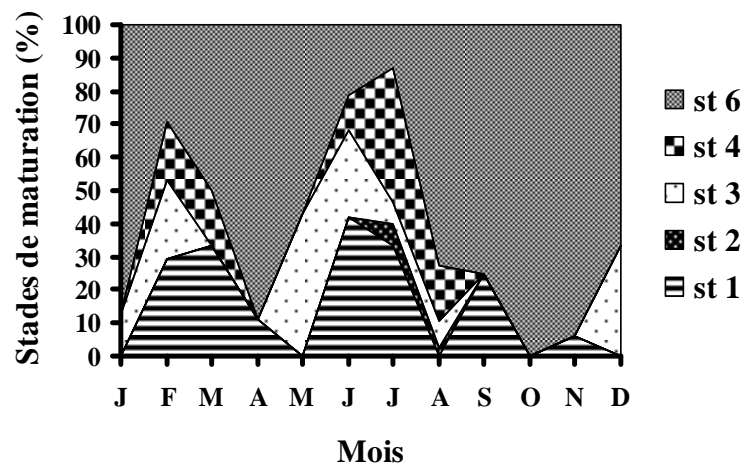

Grand- Lahou : $\quad$ A2 Mâles
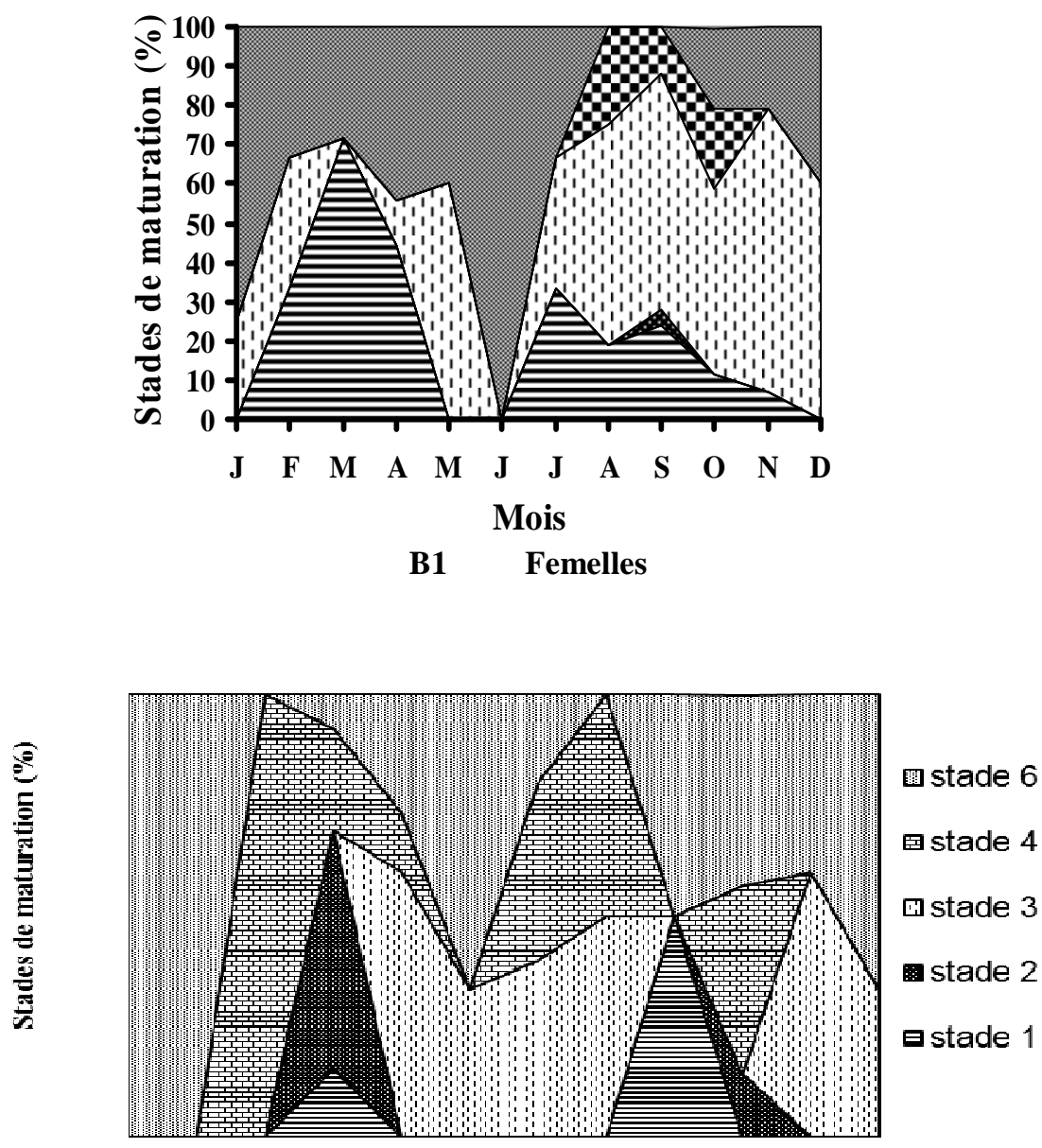

\section{Mois \\ B2 Femelles}

Figure 5 : Pourcentages de stades de maturation des mâles (A1 et A2) et femelles (B1 et B2) de cephalus respectivement dans les lagunes Ébrié et de Grand-Lahou. 


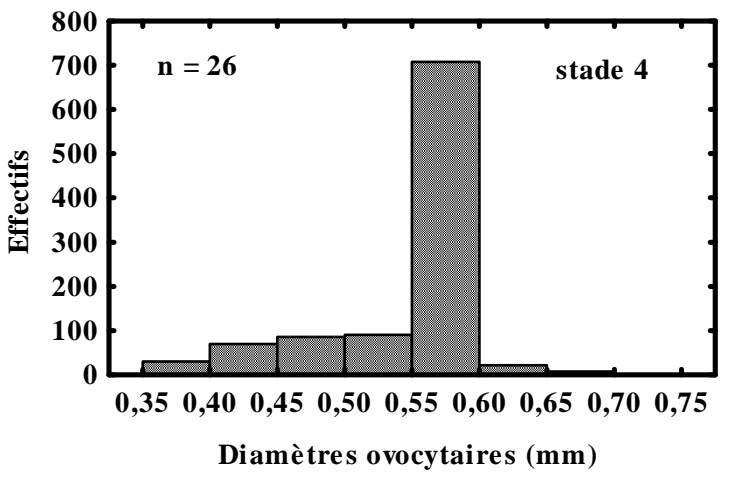

Ebrié : A1

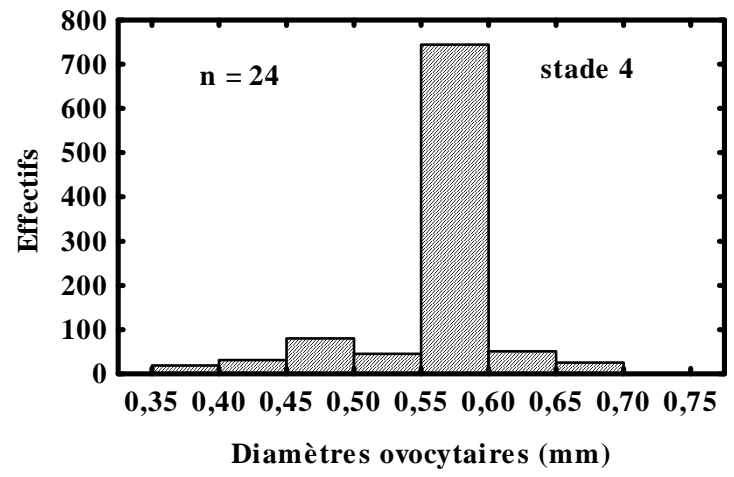

Grand-Lahou : A2

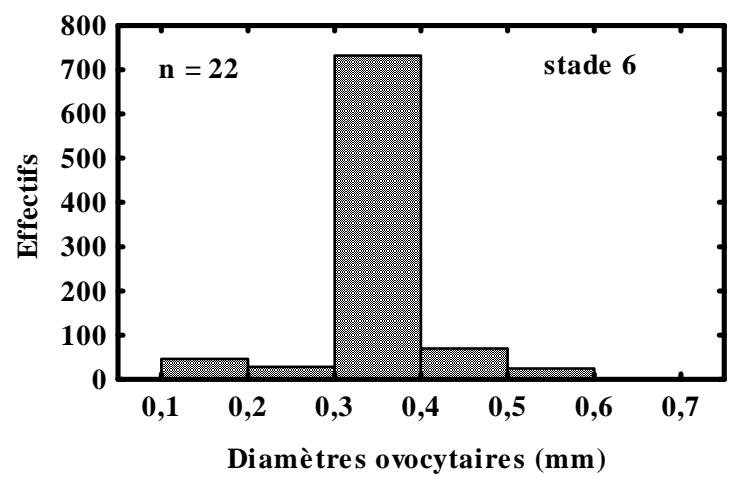

B1

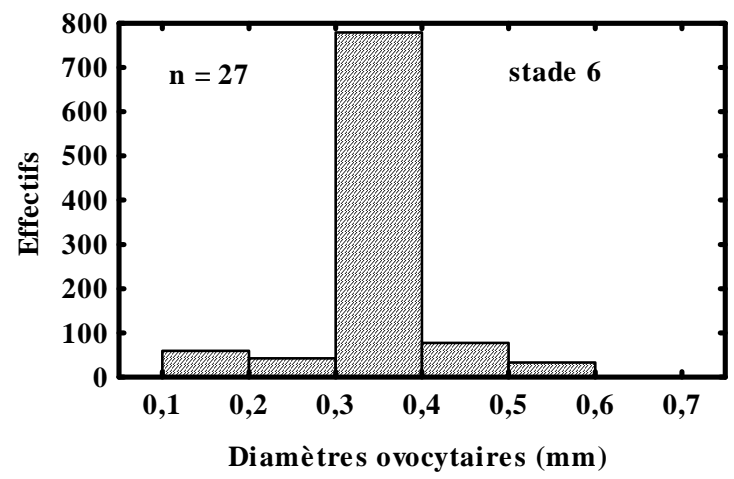

B2

Figure 6: Distribution de fréquences de tailles des ovocytes dans les gonades aux stades 4 et 6 macroscopiques chez Mugil cephalus dans les lagunes Ébrié (A1; B1) et de Grand-Lahou (A2, B2) ; $\mathrm{n}=$ nombre de femelles. 
Tableau 1: Longueur à la fourche et sex-ratio de Mugil cephalus dans les lagunes Ebrié et de Grand-lahou.

\begin{tabular}{|c|c|c|c|c|c|c|c|c|}
\hline \multirow[t]{2}{*}{ Mois } & \multicolumn{2}{|c|}{$\begin{array}{c}\text { Ebrié } \\
\text { Lmin }- \text { Lmax }(\mathbf{c m})\end{array}$} & \multicolumn{2}{|c|}{$\begin{array}{c}\text { Grand-lahou } \\
\text { Lmin - Lmax (cm) }\end{array}$} & \multirow{2}{*}{\multicolumn{2}{|c|}{$\begin{array}{c}\text { Ebrié } \\
\text { Grand-lahou }\end{array}$}} & \multirow{2}{*}{\multicolumn{2}{|c|}{$\begin{array}{c}\begin{array}{c}\text { Ebrié } \\
\text { Grand-lahou }\end{array} \\
\chi^{2}\end{array}$}} \\
\hline & Mâles & Femelles & Mâles & Femelles & & & & \\
\hline Janvier & $23,50-38,50(27)$ & $31,10-41,50(12)$ & $34,70-42,50(24)$ & $38,30-40,50(9)$ & $1: 0,44$ & $1: 0,37$ & $5,70^{*}$ & $80 *$ \\
\hline Février & $16,20-36,00(94)$ & $23,50-36,30(9)$ & $26,20-44,60(51)$ & $38,30-40,50(9)$ & $1: 0,09$ & $1: 0,18$ & $70,14 *$ & $29,40 *$ \\
\hline Mars & $18,10-48,40(73)$ & $22,50-36,20(21)$ & $37,90-40,70(18)$ & $39,40-39,40(3)$ & $1: 0,29$ & $1: 0,17$ & $28,76^{*}$ & $10,71 *$ \\
\hline Avril & $18,30-41,80(135)$ & $23,50-55,00(27)$ & $38,20-41,50(27)$ & $21,00-42,50(39)$ & $1: 0,20$ & $1: 1,44$ & $72,00^{*}$ & 2,18 \\
\hline Mai & $19,00-36,70(126)$ & $25,70-58,20(15)$ & $36,20-48,80(21)$ & $40,90-57,50(15)$ & $1: 0,12$ & $1: 0,71$ & $87,38^{*}$ & 1,00 \\
\hline Juillet & $18,00-40,00(114)$ & $23,30-33,50(9)$ & $26,1-39,90(45)$ & $36,10-52,30(15)$ & $1: 0,08$ & $1: 0,33$ & $89,63 *$ & $15,00 *$ \\
\hline Août & $25,80-37,00(132)$ & $26,40-34,20(48)$ & $21,70-34,80(37)$ & $31,40-35,90(24)$ & $1: 0,36$ & $1: 0,65$ & $39,20 *$ & 2,77 \\
\hline Septembre & $24,90-47,00(111)$ & $29,40-39,80(25)$ & $34,30-38,50(12)$ & $35,10-35,40(6)$ & $1: 0,22$ & $1: 0,50$ & $54,38 *$ & 2,00 \\
\hline Octobre & $20,50-40,00(97)$ & $22,20-38,10(44)$ & $36,90-37,60(9)$ & $23,30-38,10$ & $1: 0,45$ & $1: 2,33$ & $19,92 *$ & $4,80^{*}$ \\
\hline Novembre & $24,00-42,00(122)$ & $26,50-53,60(29)$ & $34,00-40,00(16)$ & $38,90-53,60(15)$ & $1: 24,00$ & $1: 0,94$ & $57,28 *$ & 0,03 \\
\hline Décembre & $16.50-41,00(91)$ & $30,50-37,90(15)$ & $37,20-49,00(24)$ & $31,10-49,50(9)$ & $1: 0,16$ & $1: 0,37$ & $54,49 *$ & $6,82 *$ \\
\hline
\end{tabular}

$(\mathrm{n})=$ nombre d'individus* $* *=\mathrm{p}<0,05$ 


\section{DISCUSSION}

La sex ratio observée pour l'ensemble des captures montre une prédominance des mâles de Mugil cephalus dans les lagunes Ébrié et de Grand-Lahou quelle que soit la période de l'année et la taille des individus capturés. Cette prédominance de l'un des sexes est un phénomène relativement fréquent chez de nombreuses espèces de poissons téléostéens (Poulet, 2004). Selon Paugy (1980), Santos et al. (2007) et Sylla et al. (2009) chez les téléostéens en général, les mâles sont prédominants en période de reproduction alors qu'en période de repos sexuel ce sont les femelles qui sont prédominantes. Cette hypothèse n'est pas confirmée par nos résultats puisque les mâles sont également dominants en période de repos sexuel. Fryer et Iles (1972) expliquent que la sex-ratio est en faveur des mâles parce que leur croissance est plus rapide que celle des femelles. Pour ces auteurs, la variation saisonnière de la sex-ratio pourraît être due au fait qu'une fois la fertlisation des oufs achevée, les mâles émigrent probablement des frayères vers les zones d'alimentation peu protégées où ils sont plus facilement capturés. En revanche, les femelles migrent vers les secteurs rocheux pour éviter leurs prédacteurs (y compris l'homme). D'autres hypothèses pourraient expliquer nos résultats, ce sont : la sex-ratio génétique différent de 1 ; la mortalité plus élevée des femelles aux stades larvaires et juvéniles; la moindre accessibilité des femelles dues à des différences éthologiques liées au sexe. Ces différences comportementales pourraient se traduire par une répartition plus bordière des femelles en lagune à certaines périodes de leur cycle biologique ou encore par d'éventuelles migrations vers la mer (Albaret et Légendre, 1985 ; Ameur et al., 2003). Au niveau des côtes mauritaniennes, les bancs de femelles sont plus côtiers, donc plus accessibles selon les travaux de Mohamed en 2004. En revanche, Ameur et al. (2003) et Patimar
(2008) ont signalé une dominance des femelles respectivement à l'embouchure de la lagune Merja zerga avec l'Océan Atlantique et en mer Caspiaenne.

Les mâles de Mugil cephalus atteignent la maturité sexuelle plus précocement que les femelles. Cela paraît être le cas général chez les Mugilidae (Albaret et Légendre, 1985; Ameur et al., 2003 ; Mohamed 2004). Cette précocité est liée au taux de croissance différentielle entre les mâles et femelles (Abou Seedo et Dadzie, 2004). Des résultats similaires ont été rapportés chez d'autres espèces de Mugilidae comme Liza ramada (Ergene, 1998); Liza aurata (Ilkyaz et al., 2005) et Liza saliens (Katselis et al., 2002; Patimar, 2008).

Le nombre élevé d'individus au stade 6 de maturation sexuelle par rapport au nombre d'individus au stade 4 et l'absence d'individus au stade 5 permettent de suggérer que Mugil cephalus ne réalise pas de ponte en lagune. Plusieurs auteurs s'accordent à dire que la reproduction de Mugil cephalus s'effectue en mer (Albaret et Legendre, 1985; Ameur, 1994 ; Matthieu et Mohamed, 2002 ; Ameur et al., 2003; Mohamed, 2004). Ameur et al. (2003) explique que la salinité joue un rôle prépondérant dans le processus de reproduction de Mugil cephalus. Elle serait le facteur primordial de la migration en mer car son augmentation favoriserait l'adaptation physiologique, liée à l'osmorégulation qui s'opère pendant le changement du milieu (Mohamed, 2004; Ameur et al., 2003; Cardona, 2006). Par ailleurs, Ameur et al. (2003) ont montré que d'autres facteurs comme la température et la photopériode bien que n'intervenant pas de façon directe dans le déclenchement de la période de ponte, seraient des facteurs permissifs. En effet, l'action de la température favoriserait l'augmentation de l'activité métabolique requise pour la maturation des gamètes et les transformations physiologiques qui accompagnent cette maturation (Ameur, 1994). L'évolution de 
l'IGS couplée aux variations des stades macroscopiques de maturation sexuelle montrent que la période de ponte est étalée sur toute l'année avec un pic plus marqué en Octobre. D'Août à Octobre, les gonades de Mugil cephalus acquièrent progressivement leur maturité. Les poissons s'alimentent activement et stockent des réserves énergétiques au niveau du foie et du muscle. Par conséquent, les valeurs maximales de l'IGS et $\mathrm{K}$ sont atteintes en Octobre. En revanche, l'IHS est à son plus bas niveau. L'émission des gamètes a lieu d'Octobre à janvier. Durant cette période, les poissons s'alimentent de moins en moins et commencent à puiser dans leurs réserves hépatiques et corporelles pour assurer les besoins énergétiques. Par conséquent, l'IGS et $\mathrm{K}$ sont au plus bas niveau. Cette stratégie de reproduction a été signalée chez Liza aurata des côtes Est et Sud de la Tunisie (Bedoui al., 2002). En effet, l'augmentation de l'IGS et $\mathrm{K}$ au cours de la période de maturation s'explique en partie par l'utilisation des réserves hépatiques comme source d'énergie pour la vitéllogénèse. De Février à Juin, l'IGS est à son niveau le plus bas. Cette période coïncide avec le repos sexuel. Au cours de cette période, les poissons reprennent leur alimentation ; l'IGS et $\mathrm{K}$ sont alors maintenus à des niveaux élevés. Selon Koné (2000), tenant compte du site où se réalise ce stockage (réserves énergétiques), deux types de poissons peuvent être distingués. Les poissons maigres dont les réserves s'accumulent surtout au niveau du foie. Dans ce cas, le maximum de l'IGS précède celui de l'IHS. Les poissons gras chez lesquels, les réserves sont essentiellement stockées dans les muscles, les mésentères péri-viscéraux et sous la peau. Ces réserves passent simultanément dans le foie et dans les gonades pour assurer les besoins énergétiques en période de reproduction. Pour cette catégorie de poisson, le maximum de l'IGS coïncide avec lui de l'IHS. Mugil cephalus est donc un poisson maigre puisque
l'IGS précède celui de l'IHS. La fécondité augmente avec la taille des femelles. En général, la fécondité est très élevée chez les Mugilidae mais particulièrement chez Mugil cephalus. Mc Donough et al. (2003) et Meseda et Samira (2006) ont rapporté, chez Mugil cephalus, des fécondités comprises entre $2,13.10^{5}$ et $3,01.10^{6}$ ovocytes pour des femelles de longueur totale (LT) comprises entre 33,1 et 59,2 cm. De même, Matthieu et Mohamed (2002) ont rapporté chez Mugil cephalus, des fécondités de 5 à 7 millions d'ovocytes sur les côtes Mauritaniennes. Pour Mugil cephalus qui ne pratique pas la protection parentale (Mellinger, 2002 ; AbouSeedo et Dadzie, 2004 ; Sylla et al., 2009), cette forte fécondité serait une stratégie pour maximiser la survie de la progéniture.

\section{Conclusion}

La présente étude a permis de montrer que la sex-ratio de Mugil cephalus est en faveur des mâles quelle que soit la lagune. La taille de première maturité n'est pas significativement différente entre les deux sexes dans les deux lagunes. La période d'émission des gamètes s'étend d'octobre à janvier et Mugil cephalus ne réalise pas de ponte dans les lagunes Ébrié et de GrandLahou. La distribution unimodale des diamètres ovocytaires suggère un développement synchrone des ovocytes avec une ponte unique. La forte fécondité absolue, de 126681 à 2844890 ovocytes pourrait être considérée une stratégie de reproduction pour maximiser la survie des juvéniles.

\section{REFERENCES}

Abou-Seedo F, Dadzie S. 2004. Reproductive cycle in the male and female grey mullet, Liza klunzingeri in the kuwaiti waters of the Arabian gulf. Cybium, 28(2): 97-104.

Albaret JJ. 1992. Mugilidae. In Faune des Poissons d'Eaux Douces et Saumâtres d'Afrique de l'Ouest, Lévêque C, Paugy D, Teugels GG (éds). Faune Tropicale, ${ }^{\circ}$ 
XXVIII. ORSTOM / MRAC : Paris; 780788 .

Albaret JJ, Legendre M. 1985. Biologie et écologie des Mugilidae en lagune Ébrié (Côte d'Ivoire): Intérêt potentiel pour l'aquaculture lagunaire. Rev. Hydrobiol. Trop., 18(4): 281-303.

Ameur B. 1994. Aspects biologiques et dynamiques de la population de Mugil cephalus (Poissons, Mugilidés) de la lagune de Moulay Bousselham (Maroc). Thèse de Doctorat de $3^{\text {ème }}$ cycle, Université Mohammed V, Fac. Sci. Rabat, 93 p.

Ameur B, Bayed A, Benazzou T. 2003. Rôle de la communication de la lagune de Merja Zerba (Gharb, Maroc) avec l'océan Atlantique dans la reproduction d'une population de Mugil cephalus L. (Poisson Mugilidae). Bull. Inst. Sci., Rabat, section Sciences de la Vie, 25: 77-82.

Behoui RF, Gharbi H, Abed AE. 2002. Période de reproduction et maturité sexuelle de Liza aurata (Poisson, Mugilidae) des côtes est et sud tunisiennes. Bull. Inst. Natn. Scien. Tech. Mer de Salammbô, 29: 11-15.

Cardona L. 2006. Habitat selection by grey mullets (osteichthyes: Mugilidae) in Mediterranean estuaries: the role of salinity. Scientia Marina, 70(3): 443-455.

Durand JR, Guiral D. 1994. Hydroclimat et hydrochimie. In Environnement et Ressources Aquatiques de Côte d'Ivoire, (Tome II- Les milieux lagunaires), Durand JR, Dufour P, Guiral D, Zabi SG (éds). Edition de l'ORSTOM : Paris; 129-136.

Ergene S. 1998. Reproduction characteristics of thinlip grey mullet, Liza ramata (Risso 1826) Inhabiting Akgöl-Paradeniz lagoons (Göksü Delta). Turk J. Zool., 24: $159-164$.

Ghorbel M, Jarboui O, Bradai MN, Bouain A. 1996. Détermination de la taille de première maturité sexuelle par une fonction logistique chez Limanda

limanda, Pagellus erythrinus et Scorpaena porcus, Bull. INSTM, 3: 2427.

Ilkyaz AT, Firat K, Saka S, Kinacigil HT. 2005. Age, growth, and sex ratio of golden grey Mullet, Liza aurata (Risso, 1810) in Homa lagoon (Izmir bay, Aegean sea). Turk J. Zool. 30: 279 - 284.

Katselis G, Koutsikopoulos C, Kaspiris P. 2002. Age determination and growth of leaping mullet, (Liza saliens R. 1810) from the Messolonghi Etoliko lagoon (Western Greece). Medit. Mar. Sci., 3/2: $147-148$.

Konan SK, Kouassi AM, Adingra AA, Dongui BK, Gnakri D. 2008. Variations saisonnières des paramètres abiotiques des eaux d'une lagune tropicale: la lagune de Grand Lahou, Côte d'Ivoire. European Journal of Scientific Research, 21(3): 376-393.

Koné T. 2000. Régime alimentaire et reproduction d'un tilapia lagunaire (Sarotherodon melanotheron (Rüppel, 1852) dans la rivière bia et le lac de barrage d'ayamé (Côte d'Ivoire). Thèse de Docteur en Sciences, Katholieke Universteit Leuven, Belgique, 253 p.

Magdy ME. 2004. Reproductive biology of Mugil seheli (Family Mugilidae) reared in fish farm. Egypt. J. Aquatic Res. 30(B): $234-240$.

Matthieu B, Mohamed MOV. 2002. Le Mulet en Mauritanie: biologie, écologie, pêche et aménagement. Programme Régional de Conservation de la zone côtière et Marine; $53 \mathrm{p}$.

Mc Donough CJ, Roumillat WA, Wenner CA. 2003. Fecundity and spawning season of striped mullet (Mugil cephalus L.) in South Carolina estuaries. Fish. Bull., 101: 822-834.

Mellinger J. 2002. Sexualité et Reproduction des Poissons. CNRS Editions: Paris; $349 \mathrm{p}$. 
Meseda MEG, Samira AS. 2006. Spawning induction in the Meditterranean grey mullet Mugil cephalus and larval developpemental stages. Afr. J. Biotechnol., 5(19): 1836 - 1845.

Mohamed MOV. 2004. Etude de la dynamique des systèmes d'exploitation et de l'éco-biologie de la reproduction de trois Mugilidés: Mugil cephalus (Linnaeus, 1758), Liza aurata (Perguria, 1892) et Mugil capurrii (Risso, 1810), analyse de leurs stratégies d'occupations des secteurs littoraux mauritaniens et de leurs possibilités d'aménagement. Thèse de Doctorat, Université de Nice-Sophia Antipolis, France, 129 p.

Ntakimazi G, Nzigidahera B, Nicayenzi F, West K. 2000. Etude spéciale de biodiversité (ESBIO) Rapport. L'état de la diversité biologiquedans les milieux aquatiques et terrestres du delta de la Rusizi. 70 p.

Patimar R. 2008. Some biological aspects of the sharpnose mullet Liza saliens (Risso, 1810) in Gorgan bay-Miankaleh Wildlife Refuge (the southeast Caspian sea). Turk. J. Fish. Aquat. Sci., 8: 225 - 232.

Paugy D. 1980. Ecologie et biologie des Alestes nurse (Pisces Characidae) des rivières de Côte d'Ivoire. Cah.
ORSTOM Ser. Hydrobiol., 13(3-4): 143 149.

Poulet N. 2004. Le sandre (Sander lucioperca L.) : Biologie, comportement et dynamique des populations en Camargue (Bouches de Rhône, France). Thèse de Doctorat, Université de Toulouse III, France. 300 p.

Santos JNS, Araùjo FG, Silva MA, Vasconcellos RM. 2007. Sex ratio and sexual dimorphism of the Anchovy anchoa januaria (Actinopterygii, Engraulidae) in a tropical bay in southeastern Brazil. J. Fish Biol., 71: 877-888.

Séret B, Opic P. 1986. Poissons de Mer de L'Ouest Africain Tropical. InitiationsDocumentations Techniques 49 (réédition de 1981). ORSTOM : Paris; 450 p.

Sylla S, Atsé BC, Kouassi NJ. 2009. Stratégie de reproduction du Carangidae Trachinotus teraia Cuvier, 1832 dans la lagune Ebrié (Côte d'Ivoire). Sci. Nat., 6(1): 83 - 94 . 